\title{
Electrical discharge dressing and its influence on metal bonded diamond wheels
}

\author{
Journal Article \\ Author(s): \\ Weingartner, Eduardo; Roth, Raoul; Kuster, Friedrich; Boccadoro, Marco; Fiebelkorn, Frank \\ Publication date: \\ 2012 \\ Permanent link: \\ https://doi.org/10.3929/ethz-a-007569005 \\ Rights / license: \\ In Copyright - Non-Commercial Use Permitted \\ Originally published in: \\ CIRP Annals 61(1), https://doi.org/10.1016/j.cirp.2012.03.049
}




\title{
Electrical discharge dressing and its influence on metal bonded diamond wheels
}

\author{
Eduardo Weingärtner ${ }^{\mathrm{a}}{ }^{*}$, Raoul Roth $^{\mathrm{a}}$, Friedrich Kuster (3) ${ }^{\mathrm{a}}$, Marco Boccadoro (3) ${ }^{\mathrm{b}}$, Frank Fiebelkorn ${ }^{\mathrm{c}}$ \\ ${ }^{a}$ Institute of Machine Tools and Manufacturing (IWF), ETH Zurich, Switzerland \\ ${ }^{b}$ GF AgieCharmilles, Losone, Switzerland \\ ${ }^{c}$ Fritz Studer AG, Steffisburg, Switzerland
}

Submitted by G. Levy (1), St. Gallen, Switzerland

Although EDM is a thermal removal process, when it is applied for dressing diamond grinding wheels, usually little or no thermal damage is caused to the diamonds. In this work, a better explanation for this fact is provided. A thermo-electrical model is used to calculate the temperature distribution inside diamonds showing that even for high discharge energies small amount of graphitization occur. Here, the exceptional properties of diamond contribute to minimize thermal damages. Still, a concentration of discharges can occur around the diamonds and thus lead to thermal damages. However, this phenomenon is more evident for large diamonds.

Wire EDM, Dressing, Diamond.

\section{Introduction}

Diamond is a material with exceptional properties [1]. It is an electric insulator, transparent, excellent heat conductor and known as the hardest material found in nature. For this reason, diamonds find several applications in different fields, ranging from optical to machining processes [2]. One particular application is found in grinding high performance ceramics. Metal bounded diamond wheels are widely applied in this case, since it perfectly combines the hardness of diamond grains with the wear resistance of metal bonds $[3,4]$. When it comes to conditioning, however, the above mentioned advantages are turned into disadvantages. Metal bounded diamond wheels are known as difficult-to-dress tools [5]. To overcome this problem, different dressing methods have been proposed, e.g. die-sinking and wire electrical discharge machining, which can be used for dressing metal bonded diamond wheels, since the electrically conductive bond is removed and the diamonds, which are electric insulators, cannot be eroded [4,5]. This selective removal process is of great importance for grinding, since the dressing process has to provide the grinding wheel with sufficient grain protrusion and chip space, which is important for collecting ground chips and for improving the cutting fluid transportation through the grinding zone.

Electrical discharge machining is a thermal removal process, in which discharges with highly concentrated energies warm-up, melt and evaporate material from the workpiece [6]. Depending on the discharge energy, craters with diameters up to several hundreds of micrometres are generated. When EDM is applied for conditioning metal bounded diamond wheels, one would expect thermal damages to the diamonds. Diamond is not the thermodynamically stable form of carbon at standard conditions for temperature and pressure, but rather graphite [2]. Graphite, in contrast to diamond, is a good electric conductor, widely used as electrode in die-sinking EDM, and soft, being easily machined by standard machining operations. The graphitization process, i.e. the transformation of diamond to graphite, in air, already starts at temperatures around $700{ }^{\circ} \mathrm{C}$ [2]. Klink et al. [7] measured a graphite layer of about $0.5 \mu \mathrm{m}$ on diamond grains after wire electrical discharge dressing in deionised water. In spite of that, different studies have shown that electric discharge machining is a suitable dressing process for metal bounded diamond wheels. Weingärtner et al. [4] stated that, in comparison to SiC-dressed diamond wheels, lower grinding forces are generated and better $G$-ratios are achieved when a wire electrical discharge dressed wheel was applied for grinding silicon nitride workpieces. Rhoney et al. [8] also successfully applied wire electrical discharge machining for conditioning metal bonded diamond wheels for grinding ceramics.
Although several studies have already addressed the electrical discharge dressing process, none of them really explained why little or no thermal damage occurs to the diamonds. This study aims to better understand this phenomenon. A model is used to predict which amount of graphitization is expected for different types of typical WEDM pulses, assuming that the energy of a discharge is transmitted only to a single diamond. Moreover, a discussion about the concentration of discharges around the diamond grains is presented.

\section{Influence of EDM on diamond grains}

When it comes to the process of graphitization of diamonds, different aspects have to be considered. Although graphitization can already start when a diamond is heated over $700{ }^{\circ} \mathrm{C}$ in air, Berman and Simon [9] pointed out that the conversion of diamond to graphite, at zero pressure and in an inert environment, is not detectable experimentally for temperatures below $1500{ }^{\circ} \mathrm{C}$. Davies and Evans [10] measured graphitization rates at different temperatures, and obtained activation energies of $728 \pm 50 \mathrm{KJ} / \mathrm{mol}$ and $1159 \pm 75 \mathrm{KJ} / \mathrm{mol}$, respectively for diamond surfaces $\{110\}$ and $\{111\}$. Besides that, the thermal conductivity of diamond is another important aspect. At room temperature, it can be as large as five times the thermal conductivity of copper. This has to be taken into consideration, since the heat generated by a discharge, for example, can be quickly dissipated to the whole diamond, and stays not highly concentrated at the surface, as for other materials with lower thermal conductivities.

These aspects are considered for a thermo-electric model which is used in this work to calculate the temperature distribution inside a diamond due to the energy of different erosion discharges. First, an approach to estimate which amount of discharge energy is transmitted to the workpiece/anode is presented. A so called "erosion power factor" $F_{c}$ is calculated based on a comparison between single discharge experiments and simulation results in copper, and is later used as input for further simulations with diamonds.

\subsection{Energy distribution to the workpiecelanode}

The model used to estimate the energy distribution is based on a heat conduction problem, where the temperature distribution is solved using a finite-difference method. The heat source is modelled as a time-dependent heat flux, which depends on the discharge current, discharge voltage and plasma channel diameter. Thermo-dependent material properties were taken into account, as well as the latent heat of melting and vaporization.

To estimate the erosion power factor $F_{c}$, the total volume of molten material per discharge has to be evaluated. Figure 1 
illustrates a crater eroded in copper in a single discharge experiment and a schematic representation of its cross section. The total molten volume is composed of the white layer below the reference plane plus the crater volume, which is represented as the eroded volume below the reference plane. The resolidified volume is drawn above the reference plane, and is not considered since it represents ejected material from the crater which resolidified and stayed adhered to the workpiece. The effective volume removal per crater can thus be represented by the crater volume minus the mentioned resolidified material. The volume of different craters was measured using an optical 3D measurement device Alicona InfiniteFocus. The white layer thickness was estimated according to measurements, and a value of $3 \mu \mathrm{m}$ was used as reference for the model.

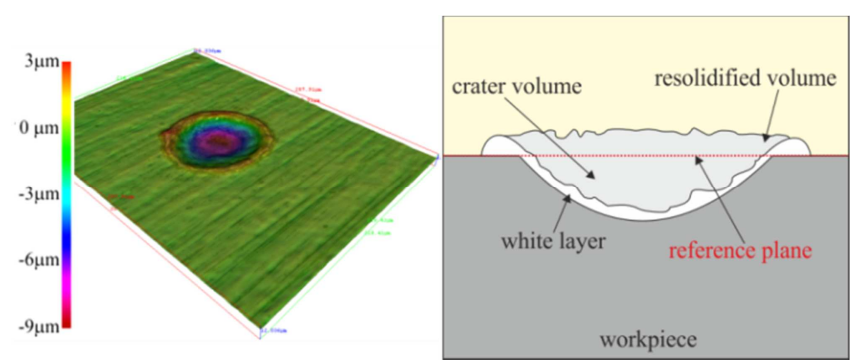

Figure 1. 3D-measurement of eroded crater (left) and schematic representation of its cross section (right).

Single discharge experiments were carried out using a Wire Cut EDM machine type AC Progress VP4 from GF AgieCharmilles. The peak current $I_{\text {peak }}$ and discharge duration $t_{e}$ used on these experiments were measured using an oscilloscope type LeCroy WaveRunner 44MXi-A and are presented in Table 1, as well as the radius of measured craters generated by these discharge pulses. The simulated cross sections of two different craters are shown in Figure 2 (workpiece of $200 \mu \mathrm{m}$ x $75 \mu \mathrm{m}$ ). The dark read area represents the region where the calculated temperature $T$ exceeded the melting temperature of copper $\left(T_{\text {melt }}=1357 \mathrm{~K}\right)$. For a peak current of $I_{\text {peak }}=88$ A (I1) the crater is shallower and has a smaller radius in comparison to $I_{\text {peak }}=223$ A (I6).

Table 1

Erosion parameters and model inputs

\begin{tabular}{|lcccccc|}
\hline \multicolumn{1}{|c}{ Model inputs } & \multicolumn{7}{c|}{ Measured values (6 different pulses) } \\
\hline & I1 & I2 & I3 & I4 & I5 & I6 \\
\cline { 2 - 7 } & 88 & 100 & 128 & 152 & 188 & 223 \\
Peak current, $I_{\text {peak }}[\mathrm{A}]$ & 1.00 & 1.05 & 1.25 & 1.35 & 1.55 & 1.80 \\
Discharge duration, $t_{e}[\mu \mathrm{s}]$ & 39 & 43 & 50 & 56 & 63 & 75 \\
Crater radius, $r_{c}[\mu \mathrm{m}]$ & & & & & & \\
\hline
\end{tabular}

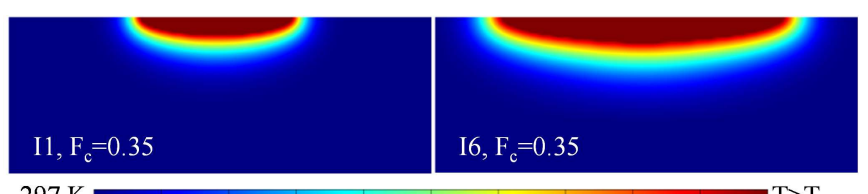

$297 \mathrm{~K}$

Figure 2. Simulated cross section of eroded craters in copper using two different peak currents (I1 and I6).

Regarding the amount of molten material per single discharge, Figure $\mathbf{3}$ shows a comparison between experimental and simulation results. The simulations were performed using power factors of $F_{c}=0.30,0.35$ and 0.40 , meaning that, respectively, $30 \%, 35 \%$ and $40 \%$ of the whole discharge energy is transmitted to the workpiece/anode. According to the presented results, a power factor of $F_{c}=0.35$ was chosen for performing further simulations with diamonds. One can see that the simulation results using $F_{c}=0.35$ don't match the experimental results for all peak currents. The reason for that lies in the choice of an equal white layer thickness for all discharge currents, which actually increases as discharge energies are increased. The choice of an erosion power factor of $F_{c}=0.35$ is also supported by the work carried out by Xia et al. (cited in Kunieda et al. [6]), who stated that in die-sinking EDM approximately $34 \%$ of the discharge energy goes to the workpiece.

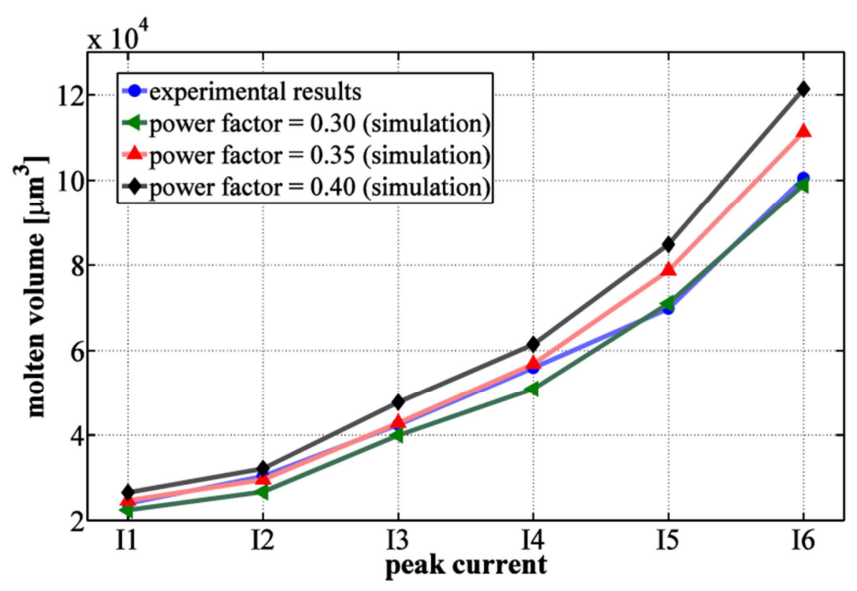

Figure 3. Determination of erosion power factor $F_{c}$.

\subsection{Heat source applied to diamond grains}

Assuming the same heat source used before, and a power factor of $F_{c}=0.35$, the temperature distribution inside single diamonds were calculated. The thermal properties of diamond were chosen to be constant, not dependent on the temperature. As model inputs, the following properties were considered: thermal conductivity $k_{c}=2000 \mathrm{~W} / \mathrm{mK}$ [2]; specific heat at constant pressure $c_{p}=509 \mathrm{~J} / \mathrm{kgK}$; mass density $\rho=3500 \mathrm{Kg} / \mathrm{m}^{3}$; activation energy of 728 and $1159 \mathrm{KJ} / \mathrm{mol}$ [10] (diamond into graphite), transition temperature $T_{g}=1800 \mathrm{~K}$.

A diamond of size D46, approximated as a cube of length 46 $\mu \mathrm{m}$, was used as a base for the model. The heat source was assumed to be a disc heat source of diameter equals the length of the cube, although, as shown in Table 1, the diameter of eroded craters are larger than that. This was assumed aiming to apply the whole discharge energy directly to the diamond. The heat source was applied on the top surface of the cube while the five other surfaces were considered to be adiabatic.

The same pulse discharges shown in Table 1 were applied in the simulations. The cube was spatially discretized with an equal distance of $1 \mu \mathrm{m}$ between consecutive nodes $(\Delta x=\Delta y=\Delta z)$, resulting in a total of 97'336 nodes. Figure 4 shows the amount of nodes that reached enough energy for graphitization. One can see that for the highest discharge energy (I6), the volume of graphitization can reach up to $6 \%$ of the whole diamond volume. This would represent a layer of circa $2.5 \mu \mathrm{m}$ on the top side of the diamond. The possible amount of graphitization increases as discharge energies are increased and less graphitization is expected for higher activation energies and larger diamonds. 


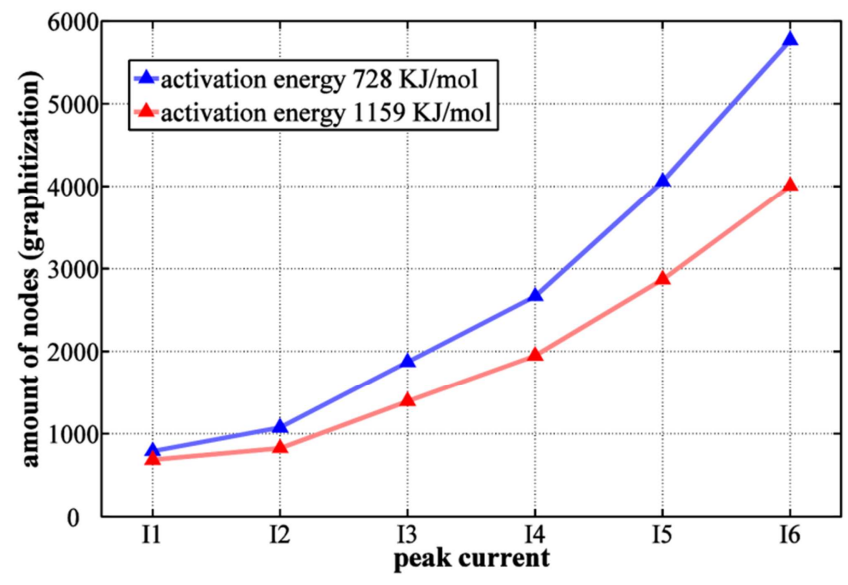

Figure 4. Simulation results of graphitization due to single discharges.

Although Figure 4 indicates the possibility of thermal damages to the diamonds, the transmission of the whole discharge energy to the diamonds, assumption made to evaluate the worst case scenario, is unlikely to happen. Diamonds are not electric conductors and, for this reason, the discharges are not likely to occur directly on the diamonds, but rather nearby. Moreover, diamonds have no adiabatic surfaces, meaning that the heat which enters the diamond can also be conducted to the bond material at the contact surfaces. Still, the plasma channel can achieve diameters larger than the diamonds, distributing the discharge energy over a larger area. For example, for a pulse type I6, craters with diameter of $150 \mu \mathrm{m}$ were measured (see Table 1), which is more than three times the size of a diamond D46. All these together contribute to the fact that electrical discharge machining processes are suitable for conditioning metal bonded diamond wheels, causing minimal thermal damages to the diamonds.

Figure 5 shows two SEM-micrographs of two metal bonded diamond grinding wheels, with diamonds sizes D46 and D91, conditioned by wire electrical discharge machining. Geometrically well-defined diamond grains with different protrusions are visible. No damages were found on these diamonds. Moreover, grinding experiments were carried out using WED-dressed and SiC-dressed grinding wheels. Silicon nitride was ground using a specific material removal rate of 10 $\mathrm{mm}^{3} /(\mathrm{mm} . \mathrm{s})$ and grinding wheel speed of $60 \mathrm{~m} / \mathrm{s}$. A higher Gratio was achieved using WED-dressed wheels $(\mathrm{G}-$ ratio=2600) in comparison to SiC-dressed wheels (G-ratio $=1900)$.

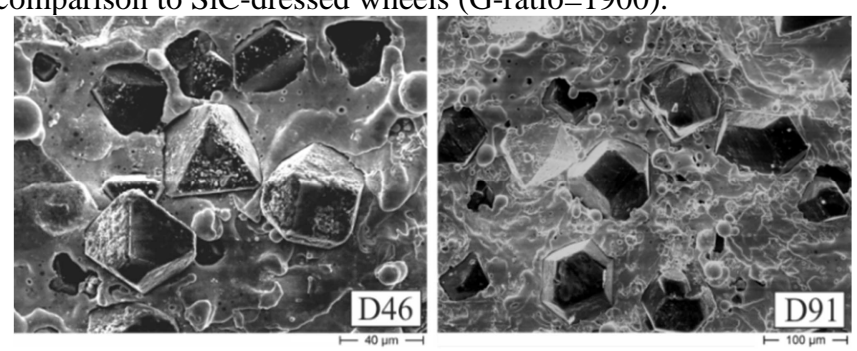

Figure 5. SEM-micrographs of WED-dressed wheels.

\section{Discharge concentration around diamond grains}

In die-sinking and wire electrical discharge machining, a stable process is achieved when the discharges are uniformly distributed over the discharge gap [11]. However, in some special cases, a concentration of discharges can occur. The dielectric strength can be weakened, for example, in locations where the gap is contaminated with eroded particles. If the electric field is concentrated in some places over the workpiece, the discharges are also more likely to occur at these locations. In a nonhomogeneous workpiece, like a grinding wheel, this phenomenon can also occur, in this case, around the diamonds. As a consequence, diamonds can be damaged or stay less strongly attached to the metal bond after erosion.

Chen and $\mathrm{Li}$ [12] investigated the anodic removal in electrolytic in-process dressing (ELID) and found that there is a electric field concentration at the diamond/metal boundary so that the metal dissolution rate is the largest at this location. Klink et al. [7] stated that, due to the different relative permittivities of medias involved in the erosion, a concentration of electric field around the diamonds is more likely to happen when deionised water is used. Moreover, they also stated that when a $\mathrm{CH}$-based dielectric is used, the electric field is concentrated on the top of the diamond grains, so that, in this case, there is a risk of high damages to the diamond. Figure 6 shows a 3D-measurement done on a metal bonded diamond pellet with diamonds of equivalent diameter 151 $\mu \mathrm{m}$ and a SEM-micrograph of the same material. This pellet was eroded in a die-sinking machine Agitron SPIRIT 2 from GF AgieCharmilles, using a $\mathrm{CH}$-based dielectric. It can be seen that much bond material was removed around the diamonds. Moreover, the SEM-micrograph revels that the diamonds have lost their original shapes, i.e. they were damaged during erosion. These surface topographies are not adequate for a grinding wheel, since the diamonds are not enough anchored to the bond, and can easily pull-out during grinding. Deionised water was also used und similar results were achieved, where even larger craters were eroded around the diamonds.

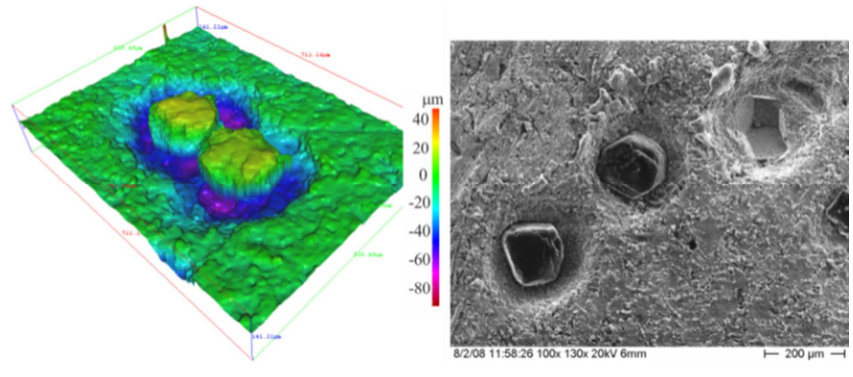

Figure 6. Concentrated erosion around diamonds after die-sinking dressing of a D151 metal bonded diamond pellet.

To evaluate this phenomenon of localized discharge concentration, the erosion of a metal bonded diamond pellet with embedded diamonds of equivalent diameter $151 \mu \mathrm{m}$ and concentration C25 was recorded using a high-speed camera. The schematic representation of this experiment is presented in Figure 7. It was carried out in a wire cut machine type Progress VP4 from GF AgieCharmilles, using deionised water as dielectric. The discharge pulse type I1 was used $\left(I_{\text {peak }}=88 \mathrm{~A}, t_{e}=1.0 \mu \mathrm{s}\right)$. The recording speed was set to thousand frames per second with an exposure time of $1000 \mu \mathrm{s}$ per frame. The erosion interval time was set to $100 \mu \mathrm{s}$, meaning that more than one discharge can be recorded per acquisition frame. The recorded frames were than edited, so that only one line is cut out of each frame (represented by $F 1$ to $F n$ in Figure 7), and put together side by side to form a sequence of discharges. Thus, a mapping of discharges is generated and can be further analysed. 

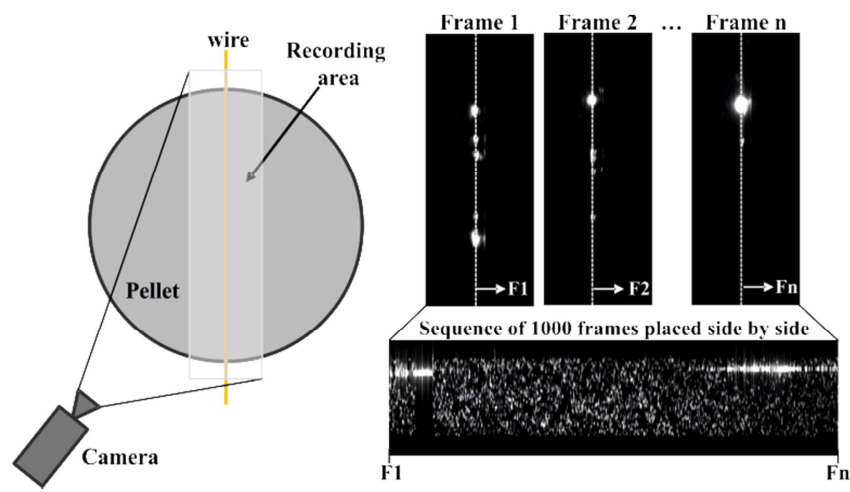

Figure 7. Representation of setup for recording discharge sequences.

Figure 8 show two discharge distribution sequences, each of which representing thousand frames, i.e. a total acquisition time of one second per sequence. A region of uniform distribution of discharges can be recognized and some regions where the discharges are concentrated are visible. The position of the wire relative to the workpiece during the acquisition was known (in $\mathrm{x}$ and $y$ direction). Thus, it was possible to relate the location of discharges with the topography of the pellet. It was found that the localized concentration of discharges happens just around the diamonds, having the consequences mentioned before.

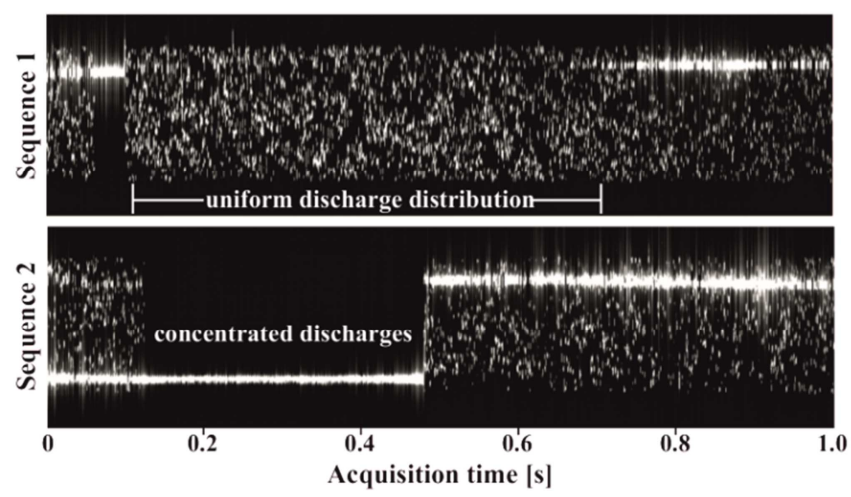

Figure 8. Discharge mapping (different discharge distribution patterns).

Although the above mentioned results represent a negative impact for WED-dressed grinding wheels, it was found that this phenomenon is more evident for larger diamonds. Grinding wheels D46, D91, D126 and D151 were evaluated, and this concentration of discharges was observed in all but the grinding wheel D46. The grinding wheels were also conditioned at speeds up to $50 \mathrm{~m} / \mathrm{s}$, and even in these cases, where two consecutive discharges cannot occur in the same location, the concentrated erosion was observed. The size of the erosion GAP was measured on a brass workpiece eroded with $I_{\text {peak }}=223$ A (I6) using grinding oil and deionized water and found to be 28 and $36 \mu \mathrm{m}$ respectively. This indicates that the erosion of grinding wheels with larger diamonds is critical. Grinding marks were visible on the wire electrode when diamonds D91 and larger were used. Thus, this dressing method is more suitable for small abrasive grains, since both, the concentrated erosion is more evident for larger diamonds and the size of the erosion gap is much smaller than these grain sizes.

\section{Conclusions}

Although high temperatures are reached in electrical discharge machining, diamond graphitization does not happen easily during electrical discharge dressing, since both a high amount of energy is necessary to overcome the energy of activation to transform diamond into graphite and diamonds have very high thermal conductivities, which help distributing the energy quickly to the whole diamond and its surrounding. The diamonds can however be damaged when a localized concentration of discharges occur around the grains. A mapping of discharge distribution showed that during the erosion of a pellet with diamonds D151, locations of highly concentrated discharges were identified. This phenomenon happens due to a stronger electric field which is formed just at the interface between diamonds and bond material. As a result, huge craters are crated around the diamonds and even thermal damages occur to these abrasives. Experimental results showed, however, that this phenomenon is more likely to occur when large diamond grains are used. The issues discussed in the paper shall be explored further, e.g. by analysis of the degradation of the diamonds due to different erosion inputs with techniques like Micro-Raman spectroscopy.

\section{Acknowledgement}

The authors would like to thank Studer AG, GF AgieCharmilles, Diametal AG, Blaser Swisslube AG, CeramTec AG, and CTI of Switzerland for their support.

\section{References}

[1] Seal M (1992) Applications exploiting the extreme properties of diamonds. Materials Science and Engineering 11:167-171.

[2] Field JE (1979) The Properties of diamond. Academic Press; 1979.

[3] Lin CS, Yang YL, Lin ST (2008) Performances of metal-bond diamond tools in grinding alumina. J. of Materials Processing Technology 201:612-617.

[4] Weingartner E, Jaumann S, Kuster F, Wegener K (2010) On-machine wire electrical discharge dressing (WEDD) of metal-bonded grinding wheels. International Journal of Advanced Manufacturing Technology 49:1001-1007.

[5] Wegener K, Hoffmeister HW, Karpuschewski B, Kuster F, Hahmann WC, Rabiey M (2011) Conditioning and monitoring of grinding wheels. Annals of CIRP 60(2):757-77.

[6] Kunieda M, Lauwers B, Rajurkar KP, Schumacher BM (2005) Advancing EDM through Fundamental Insight into the Process. Annals of CIRP 54(2):64-87.

[7] Klink A (2010) Wire electro discharge trueing and dressing of fine grinding wheels. Annals of CIRP 59(1):235-238.

[8] Rhoney BK, Shih AJ, Scattergood RO, Akemon JL, Gust DJ, Grant MB (2002) Wire electrical discharge machining of metal bond diamond wheels for ceramic grinding. Int. J. of Machine Tools and Manufacture 42:1355-1362.

[9] Berman R, Simon F (1955) On the graphite-diamond equilibrium. Zeitschrift für Elektrochemie 59:333-8.

[10] Davies G, Evans T (1972) Graphitization of Diamond at Zero Pressure and at a High Pressure. Proceedings of the Royal Society of London 328:413-27.

[11] Kunieda M, Kojima H, Kinoshita N (1990) On-Line Detection of EDM Spark Locations by Multiple Connection of Branched Electric Wires. Annals of CIRP 39(1):171-4.

[12] Chen H, Li JCM (2008) Anodic metal matrix removal rate in electrolytic inprocess dressing I: Two-dimensional modeling. Journal of Applied Physics 87:3151-8. 